\title{
Quantum State Engineering in Multi-Level Systems Using Shortcut to Adiabatic Passage
}

\author{
M. SAADATI-NIARI* AND M. KIAZAND \\ Department of Physics, Faculty of Sciences, University of Mohaghegh Ardabili, \\ P.O. Box 179, Ardabil, Iran
}

Received: 08.05.2020 \& Accepted: 28.07.2020

Doi: $10.12693 /$ APhysPolA.138.794

*e-mail: m.saadati@uma.ac.ir

\begin{abstract}
We extend the shortcut to the adiabatic passage method, proposed by Chen et al. [Phys. Rev. Lett. 105,123003 (2010)], to the creation of an arbitrary coherent superposition of states in a multi-level system. We consider a multi-state system composed of an initial ground state, an excited state and an arbitrary number of final states. In this method, the initial state is linked to the excited state using a pump laser pulse and final states are coupled to the excited state using the Stokes laser pulses. Furthermore, the initial state is coupled to the final states using additional laser fields. We show that the problem can be reduced to the solution of a three-state loop system involving an initial ground state, an excited state and a superposition of final states. This decomposition allows to create an arbitrary coherent superposition of states using the shortcut to the adiabatic passage method. When compared to other strategies, this method provides a fast and robust approach to the coherent superposition of states.
\end{abstract}

topics: coherent superposition, multi-level systems, population transfer, shortcut to adiabatic passage

\section{Introduction}

The creation of a coherent superposition of quantum states has a rich variety of applications, such as chemical processes, quantum information processing and nonlinear optics [1]. The stimulated Raman adiabatic passage (STIRAP) [2-7] and $\pi$-pulse [8] are two important quantum optics techniques used to create a coherent superposition in multi-state systems [9-12]. In recent years, STIRAP-like techniques, such as a piecewise adiabatic passage (PAP) [13-15], a train of coincident pulses [16] and a digital adiabatic passage (DAP) [17] have been very common and popular in fulfilling research expectations [18-22]. The $\pi$-pulse is a fast technique but it requires resonant pulses and therefore is highly sensitive to variations in the pulse area and to inhomogeneities in the sample [23]. Although the STIRAP method is insensitive to variations in the experimental parameters, it usually needs a relatively long interaction time. If the time required for evolution is too long, the scheme may be useless because decoherence would destroy coherent dynamics of the system.

In recent years, the acceleration of system dynamics to drive it to a desired final state has been considered and, in fact, a lot of work has been undertaken to find shortcuts to adiabaticity [24-35]. It is shown that the shortcuts to adiabaticity can be used for fast population transfer in multi-particle systems [36], mode conversion in optical waveguides $[37,38]$ and generation of the Greenberger-Horne-Zeilinger states (GHZ) [39]. The proposed method in [27] is superadiabatic STIRAP (sa-STIRAP) and based on counterdiabatic driving. The basic idea of counter-diabatic driving is to add auxiliary interactions to some reference Hamiltonian so that the dynamic follows exactly the (approximate) adiabatic evolution driven by the reference Hamiltonian. In a three-state system, an additional field is applied on the transition of ground states to form a loop linkage of the three states rather than the $\Lambda$-like linkage. This method [27] provides a fast and robust approach to population control and the time evolution of system remains on a non-adiabatic pass.

In this paper, we show that the sa-STIRAP method, hitherto used for complete population transfer amongst three-state quantum systems, offers an efficient and robust method for creating coherent superpositions of states in multi-level systems. We consider a linkage pattern composed of an initial populated ground state, an optional number of final ground states and an excited state. In our method, all ground states are paired to the excited state using the pump and the Stokes pulses. Apart from that, the initial populated ground state is paired to the final ground states using laser pulses different from the pump and the Stokes pulses. It is shown that in the appropriate conditions for 
pulse amplitudes, the proposed linkage can be reduced to a three-state loop system which consists of an initial populated ground state, a coherent superposition of final ground states and an excited state. Using the sa-STIRAP method in a reduced three-state loop, the population is transferred from the initial populated ground state to an arbitrary coherent superposition of the final ground states. Our method possesses all of the advantages that one can consider for sa-STIRAP in the three-state system such as the evolution in a short time and robustness because of parameter variations.

This paper is organized as follows. In Sect. 2, the sa-STIRAP method in a three-state system is described. Section 3 proposes a general solution to the implementation of sa-STIRAP method in a multi-level system. In Sect. 4 the pulses are designed and some numerical simulations are presented. Section 5 describes the robustness of our method due to the fluctuations in the time delay and peak Rabi frequency. Finally, Sect. 6 presents a summary of the results.

\section{Shortcut to adiabatic passage in three-state systems}

We consider a three-state system linked by two time-dependent interactions to be referred to as a pump between state $|1\rangle$ of energy $E_{1}$ and the excited state $|2\rangle$ having energy $E_{2}$, and the Stokes interaction between the intermediate state $|2\rangle$ and the final target state $|3\rangle$ having energy $E_{3}$. The Hamiltonian within the rotating wave approximation (RWA) [8, 40] reads as

$$
H_{0}^{I}(t)=\frac{\hbar}{2}\left(\begin{array}{ccc}
0 & \Omega_{P}(t) & 0 \\
\Omega_{P}(t) & 2 \Delta_{P} & \Omega_{S}(t) \\
0 & \Omega_{S}(t) & 2 \Delta_{3}
\end{array}\right),
$$

where $\Omega_{p}$ and $\Omega_{S}$ - the pump and the StokesRabi frequencies. The detunings from resonance are defined by $\Delta_{p}=\omega_{p}-\left(E_{2}-E_{1}\right) / \hbar$, $\Delta_{S}=\omega_{S}-\left(\left|E_{3}-E_{2}\right|\right) / \hbar$ and $\Delta_{3}=\Delta_{p}-\Delta_{S}$, while $\omega_{p}$ and $\omega_{S}$ are the laser frequencies of the pump and of the Stokes laser, respectively. For the transfer process of our interest, it is essential (a)

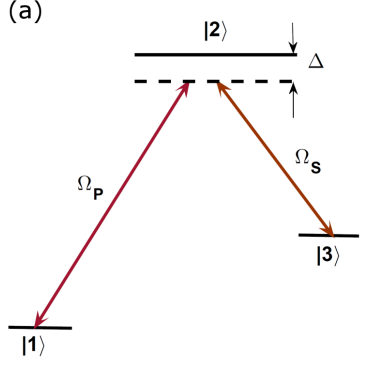

(b)

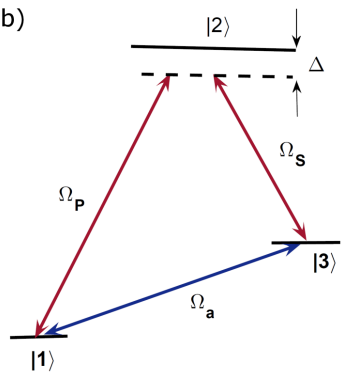

Fig. 1. (a) Level scheme of a three-state $\Lambda$-like system. (b) Level scheme of a three-state loop system in which an additional field was applied on the transition of ground states in a $\Lambda$-like system. to apply $\Delta_{3}=0$ (the two-photon resonance condition). Now, considering $\Delta_{p}=\Delta$ (see Fig. 1a), the three-level analysis can be written in an even simpler form. Namely,

$$
\begin{aligned}
& \tan (\theta)=\frac{\Omega_{p}(t)}{\Omega_{S}(t)}, \\
& \tan (2 \phi)=\frac{\Omega}{\Delta}, \quad \Omega=\sqrt{\Omega_{p}^{2}(t)+\Omega_{S}^{2}(t)},
\end{aligned}
$$

where $\theta$ and $\phi$ are the mixing angles. the Hamiltonian eigenvalues are written as

$$
\begin{aligned}
& E_{+}(t)=\hbar \Omega \cot (\phi / 2), \\
& E_{-}(t)=-\hbar \Omega \tan (\phi / 2), \\
& E_{0}=0
\end{aligned}
$$

and the instantaneous eigenvectors as [40, 41]:

$$
\begin{aligned}
& \left|\lambda_{+}(t)\right\rangle=\sin (\theta) \sin (\phi)|1\rangle+\cos (\phi)|2\rangle \\
& \quad+\cos (\theta) \sin (\phi)|3\rangle \\
& \left|\lambda_{-}(t)\right\rangle=\sin (\theta) \cos (\phi)|1\rangle-\sin (\phi)|2\rangle \\
& \quad+\cos (\theta) \cos (\phi)|3\rangle \\
& \left|\lambda_{0}(t)\right\rangle=\cos (\theta)|1\rangle-\sin (\theta)|3\rangle .
\end{aligned}
$$

The simplest Hamiltonian $H_{1}^{I}(t)$ steers the dynamics along the instantaneous eigenstates $\left|\lambda_{n}(t)\right\rangle$ of $H_{0}^{I}(t)$ without transitions among them and without phase factors. Formally, in an arbitrarily short time, it is given by [29]:

$$
\begin{aligned}
& H_{1}^{I}(t)=\mathrm{i} \hbar \sum_{n}\left|\partial_{t} \lambda_{n}\right\rangle\left\langle\lambda_{n}\right|= \\
& \mathrm{i} \hbar\left(\begin{array}{ccc}
0 & \dot{\phi} \sin (\theta) & \dot{\theta} \\
-\dot{\phi} \sin (\theta) & 0 & -\dot{\phi} \cos (\theta) \\
-\dot{\theta} & \dot{\phi} \cos (\theta) & 0
\end{array}\right)
\end{aligned}
$$

with

$$
\begin{aligned}
& \dot{\theta}=\frac{1}{\Omega^{2}}\left[\dot{\Omega}_{P}(t) \Omega_{S}(t)-\dot{\Omega}_{S}(t) \Omega_{P}(t)\right], \\
& \dot{\phi}=\frac{\Delta}{2 \Omega\left(\Delta^{2}+\Omega^{2}\right)}\left[\dot{\Omega}_{P}(t) \Omega_{P}(t)+\dot{\Omega}_{S}(t) \Omega_{S}(t)\right] .
\end{aligned}
$$

The total Hamiltonian is $H(t)=H_{0}^{I}(t)+H_{1}^{I}(t)$ and thus three new interactions should be added to the original Hamiltonian. The associated dynamics is determined by the Schrödinger equation

$$
\mathrm{i} \hbar \frac{\partial}{\partial t}|\Psi(t)\rangle=H(t)|\Psi(t)\rangle
$$

where the elements of the three-dimensional vector $|\Psi(t)\rangle$ are the probability amplitudes of the states. If the transition between the ground states is electric-dipole forbidden, a magnetic dipole transition may be used instead. When working in the adiabatic basis, the additional fields to $|1\rangle \rightarrow|2\rangle$ and $|2\rangle \rightarrow|3\rangle$ are not necessary [27] and $H_{1}^{I}(t)$ may therefore be simplified to

$$
H_{1}^{I}(t)=\frac{\hbar}{2}\left(\begin{array}{ccc}
0 & 0 & \mathrm{i} \Omega_{a} \\
0 & 0 & 0 \\
-\mathrm{i} \Omega_{a} & 0 & 0
\end{array}\right),
$$




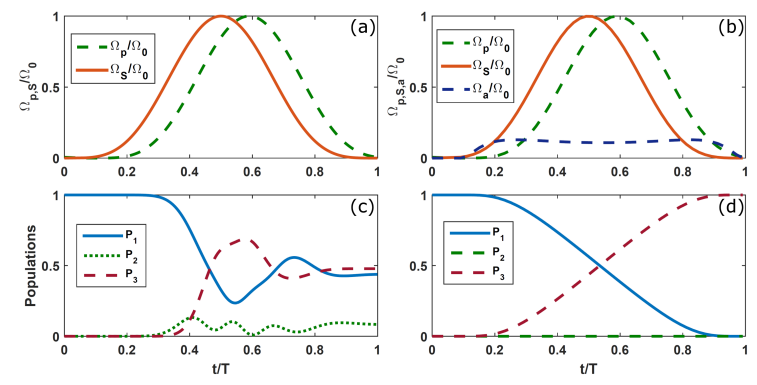

Fig. 2. Time evolution of the Rabi frequencies and time dependence of the $|1,2,3\rangle$ populations for the STIRAP $(\mathrm{a}, \mathrm{c})$ and sa-STIRAP $(\mathrm{b}, \mathrm{d})$ methods, respectively, for $\Omega_{0}=1.5 \pi \mathrm{MHz}, \Delta=0.5 \pi \mathrm{MHz}$, $T=8 \mu$ s and $\tau=0.09 T$ in all cases.

where $\Omega_{a}=2 \dot{\theta}$ (see Fig. 1b). We impose that the initial state of the system is $|1\rangle$ and also that the pulses satisfy the well-known counterintuitive sequence with $\Omega_{S}$ first and $\Omega_{p}$ later. Using these conditions, the system will first be in the dark state $\left|\lambda_{0}(t)\right\rangle$ and after using $H_{1}^{I}(t)$ the system evolution will follow exactly this eigenstate of $H_{0}^{I}(t)$. The population, therefore, will be completely transferred to $|3\rangle$. The adiabatic following $\left|\lambda_{0}(t)\right\rangle$ takes place for any choice of the protocol parameters, even with very small values of the applied pump and Stoke fields, and in arbitrarily short time. In order to perform a numerical study, the pump and Stokes pulses are considered as follows:

$$
\begin{aligned}
& \Omega_{p}(t)=\Omega_{0} f(t-\tau), \quad \Omega_{S}(t)=\Omega_{0} f(t), \\
& f(t)= \begin{cases}\sin ^{4}\left(\pi \frac{t}{T}\right) & 0<t<T, \\
0 & \text { otherwise. }\end{cases}
\end{aligned}
$$

Figure $2 \mathrm{a}$ and $2 \mathrm{c}$ shows the time evolution of the Rabi frequencies and populations using the STIRAP Stokes-pump pulse sequence, respectively. It can be seen that, by considering $\Omega_{0}=2 \pi \times 2 \mathrm{MHz}, \quad \Delta=2 \pi \times 0.1 \mathrm{MHz}, \quad T=4 \mu \mathrm{s}$ and $\tau=0.1 T$, the adiabaticity breaks down and the population is not fully transferred to the final state. Figure $2 \mathrm{~b}$ and $2 \mathrm{~d}$ shows the time evolution of the Rabi frequencies and populations, respectively, in which an additional field $\Omega_{a}(t)$ is applied on the transition of $|1\rangle \rightarrow|3\rangle$ (sa-STIRAP). The laser parameters used in all cases of Fig. 2 are the same, however the population is fully transferred to the final state in the case presented in Fig. 2d.

\section{Superadiabatic STIRAP in multi-state system}

We consider a $N+2$-state system whose linkage pattern is depicted in Fig. 3a. In our method, the ground state $|a\rangle$ is coupled via the upper state with $\Omega_{p}(t)$ and the other $N$ ground states are coupled via the upper state with $N$ independent
Stokes pulses $\Omega_{S}^{(1)}(t), \Omega_{S}^{(2)}(t), \ldots, \Omega_{S}^{(N)}(t)$. We impose the Stokes pulses to be of the same shape. In addition, we assume that the state $|a\rangle$ is coupled to the other ground states $|1\rangle,|2\rangle, \ldots,|N\rangle$ with the same shape pulses $\Omega_{a}^{(1)}, \Omega_{a}^{(2)}, \ldots, \Omega_{a}^{(N)}$. The $N+1$ lower states are coupled via the excited state $|e\rangle$ with pulsed interactions whose each pair is on two-photon resonance. The total Hamiltonian is $H(t)=H_{0}^{I}(t)+H_{1}^{I}(t)$, where $H_{0}^{I}(t)$ and $H_{1}^{I}(t)$ belong to the subspace $S=\{|a\rangle,|e\rangle,|1\rangle, \ldots,|N\rangle\}$ and in the rotating-wave approximation, they are described by:

$$
H_{0}(t)=\frac{\hbar}{2}\left(\begin{array}{cccccc}
0 & \Omega_{P} & 0 & 0 & \cdots & 0 \\
\Omega_{P} & 2 \Delta & \Omega_{S}^{(1)} & \Omega_{S}^{(2)} & \cdots & \Omega_{S}^{(N)} \\
0 & \Omega_{S}^{(1)} & 0 & 0 & \cdots & 0 \\
0 & \Omega_{S}^{(2)} & 0 & 0 & \cdots & 0 \\
\vdots & \vdots & \vdots & \vdots & \ddots & \vdots \\
0 & \Omega_{S}^{(N)} & 0 & 0 & \cdots & 0
\end{array}\right),
$$

$$
H_{1}(t)=\frac{\hbar}{2}\left(\begin{array}{cccccc}
0 & 0 & \mathrm{i} \Omega_{a}^{(1)} & \mathrm{i} \Omega_{a}^{(2)} & \ldots & \mathrm{i} \Omega_{a}^{(N)} \\
0 & 0 & 0 & 0 & \ldots & 0 \\
-\mathrm{i} \Omega_{a}^{(1)} & 0 & 0 & 0 & \ldots & 0 \\
-\mathrm{i} \Omega_{a}^{(2)} & 0 & 0 & 0 & \ldots & 0 \\
\vdots & \vdots & \vdots & \vdots & \ddots & \ldots \\
-\mathrm{i} \Omega_{a}^{(N)} & 0 & 0 & 0 & \ldots & 0
\end{array}\right) .
$$

For the sake of simplicity, the Rabi frequencies of the couplings between the ground states and the excited state $\Omega_{p}(t), \Omega_{S}^{(1)}(t), \Omega_{S}^{(2)}(t), \ldots, \Omega_{S}^{(N)}(t)$ are assumed to be real and positive as the populations do not depend on their signs. Our goal is to transfer the $N+2$-state system to a three-loop system. We impose that the pulses meet the following requirements:

$$
\begin{aligned}
& \frac{\Omega_{S}^{(2)}}{\Omega_{S}^{(1)}}=\frac{\Omega_{a}^{(2)}}{\Omega_{a}^{(1)}}=\tan \left(\eta_{1}\right), \\
& \frac{\Omega_{S}^{(3)}}{\sqrt{\left(\Omega_{S}^{(1)}\right)^{2}+\left(\Omega_{S}^{(2)}\right)^{2}}}=\frac{\Omega_{a}^{(3)}}{\sqrt{\left(\Omega_{a}^{(1)}\right)^{2}+\left(\Omega_{a}^{(2)}\right)^{2}}}=\tan \left(\eta_{2}\right), \\
& \quad \vdots \\
& \sqrt{\sum_{i=1}^{N-1}\left(\Omega_{S}^{(i)}\right)^{2}}=\frac{\Omega_{a}^{(N)}}{\sqrt{\sum_{i=1}^{N-1}\left(\Omega_{a}^{(i)}\right)^{2}}}=\tan \left(\eta_{N-1}\right),
\end{aligned}
$$

where $\eta_{1}, \eta_{2}, \ldots, \eta_{N-1}$ are the time-independent mixing angles. We emphasize that the parametrization of the mixing angles we have defined here is different from the one used in [19] in which the Morris-Shore (MS) [42-44] transformation is used step by step to reduce a coupled Hilbert space into a three-state $\Lambda$-like system. The corresponding 


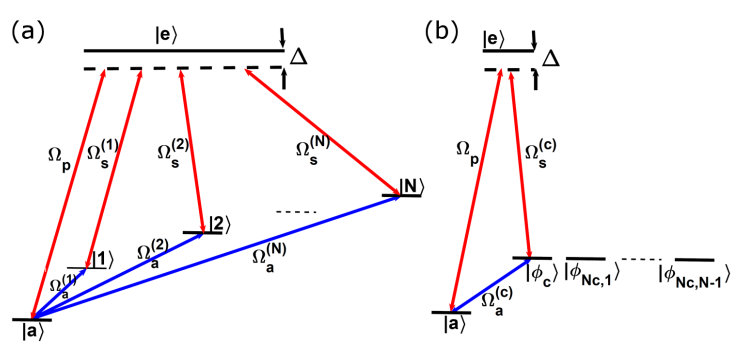

Fig. 3. (a) Linkage pattern of the $N$-pod system. (b) Linkage pattern of the $N$-pod system in the new basis.

time-independent transformation in the new bases $S^{T}=\left\{|1\rangle,|e\rangle,\left|\phi_{C}\right\rangle,\left|\phi_{N C, 1}\right\rangle,\left|\phi_{N C, 2}\right\rangle, \ldots,\left|\phi_{N C, N-1}\right\rangle\right\}$ reads as

$$
T=\left(\begin{array}{cc}
I & O \\
O & T_{b}
\end{array}\right)
$$

where $I$ indicates the two-dimensional identity matrix and $O$ is the zero matrix. In turn,

$$
T_{b}=\left(\begin{array}{ccccc}
\chi_{1} & -\frac{\chi_{2}}{X_{2}} & \frac{\chi_{1} \chi_{3}}{X_{2} X_{3}} & \cdots & \frac{\chi_{1} \chi_{N}}{X_{N-1} X_{N}} \\
\chi_{2} & \frac{\chi_{1}}{X_{2}} & \frac{\chi_{2} \chi_{3}}{X_{2} X_{3}} & \cdots & \frac{\chi_{2} \chi_{N}}{X_{N-1} X_{N}} \\
\chi_{3} & 0 & -\frac{X_{2}^{2}}{X_{2} X_{3}} & \cdots & \frac{\chi_{3} \chi_{N}}{X_{N-1} X_{N}} \\
\vdots & \vdots & \vdots & \ddots & \vdots \\
\chi_{N} & 0 & 0 & 0 & \frac{-X_{N-1}^{2}}{X_{N-1} X_{N}}
\end{array}\right)
$$

with

$$
\begin{aligned}
& \chi_{1}=C_{1} C_{2} \ldots C_{N-1}, \\
& \chi_{2}=S_{1} C_{2} \ldots C_{N-1}, \\
& \vdots \\
& \chi_{N}=S_{N-1},
\end{aligned}
$$

where $S_{j}=\sin \left(\eta_{j}\right)$ and $C_{j}=\cos \left(\eta_{j}\right)$ and $X_{l}$ is given by

$$
X_{l}=\sqrt{\sum_{k=1}^{l}\left(\chi_{k}^{2}\right)}, \quad(l=1,2,3, \ldots, N) .
$$

The transformation (20) allows for the construction of an equivalent system featuring a three-state loop system in the subspace $\left\{|a\rangle,|e\rangle,\left|\phi_{C}\right\rangle\right\}$ with the coupled state

$$
\left|\phi_{C}\right\rangle=\chi_{1}|1\rangle+\chi_{2}|2\rangle+\cdots+\chi_{N}|N\rangle
$$

and $N-1$ uncoupled states in the subspace $\left\{\left|\phi_{N C, 1}\right\rangle,\left|\phi_{N C, 2}\right\rangle, \ldots,\left|\phi_{N C, N-1}\right\rangle\right\}$ such that

$$
\begin{aligned}
& \left|\phi_{N C, 1}\right\rangle=-\frac{\chi_{2}}{X_{2}}|1\rangle+\frac{\chi_{1}}{X_{2}}|2\rangle \\
& \left|\phi_{N C, 2}\right\rangle=\frac{\chi_{1} \chi_{3}}{X_{2} X_{3}}|1\rangle+\frac{\chi_{2} \chi_{3}}{X_{2} X_{3}}|2\rangle-\frac{X_{2}^{2}}{X_{2} X_{3}}|3\rangle, \\
& \vdots \\
& \left|\phi_{N C, N-1}\right\rangle=\frac{\chi_{1} \chi_{N}}{X_{N-1} X_{N}}|1\rangle+\frac{\chi_{2} \chi_{N}}{X_{N-1} X_{N}}|2\rangle \\
& +\frac{\chi_{3} \chi_{N}}{X_{N-1} X_{N}}|3\rangle+\cdots-\frac{X_{N-1}^{2}}{X_{N-1} X_{N}}|N\rangle .
\end{aligned}
$$

The transformed Hamiltonian $H^{T}(t)=T^{\dagger} H(t) T$ in the new bases $\left\{|a\rangle,|e\rangle,\left|\phi_{C}\right\rangle\right\}$ reduced to a threestate system reads as follows:

$$
H^{T}(t)=\frac{\hbar}{2}\left(\begin{array}{ccc}
0 & \Omega_{P} & \mathrm{i} \Omega_{a}^{(C)} \\
\Omega_{P} & 2 \Delta & \Omega_{S}^{(C)} \\
-\mathrm{i} \Omega_{a}^{(C)} & \Omega_{S}^{(C)} & 0
\end{array}\right) .
$$

The linkage pattern in the new bases is presented in Fig. 3b.

The Hamiltonian (26) can be written as $H^{\mathrm{T}}(t)=H_{0}^{\mathrm{T}}(t)+H_{1}^{\mathrm{T}}(t)$, where

$$
H_{0}^{\mathrm{T}}(t)=\frac{\hbar}{2}\left(\begin{array}{ccc}
0 & \Omega_{p} & 0 \\
\Omega_{p} & 2 \Delta & \Omega_{S}^{(C)} \\
0 & \Omega_{S}^{(C)} & 0
\end{array}\right)
$$

and

$$
H_{1}^{\mathrm{T}}(t)=\frac{\hbar}{2}\left(\begin{array}{ccc}
0 & 0 & \mathrm{i} \Omega_{a}^{(C)} \\
0 & 0 & 0 \\
-\mathrm{i} \Omega_{a}^{(C)} & 0 & 0
\end{array}\right) .
$$

Here,

$$
\begin{aligned}
& \Omega_{S}^{(C)}=\sqrt{\sum_{i=1}^{N}\left(\Omega_{S}^{(i)}\right)^{2},} \\
& \Omega_{a}^{(C)}=\sqrt{\sum_{i=1}^{N}\left(\Omega_{a}^{(i)}\right)^{2} .}
\end{aligned}
$$

Next, we consider that the reduced Hamiltonian $H_{1}^{\mathrm{T}}(t)$ in the new basis $S=\left\{|a\rangle,|e\rangle,\left|\phi_{C}\right\rangle\right\}$ steers the dynamics along the instantaneous eigenstates $\left|\lambda_{n}^{\mathrm{T}}(t)\right\rangle$ of $H_{0}^{\mathrm{T}}(t)$ without transition among them and without phase factors. The instantaneous eigenstates $\left|\lambda_{n}^{\mathrm{T}}\right\rangle$ of $(27)$ are

$$
\begin{aligned}
& \left|\lambda_{+}^{\mathrm{T}}(t)\right\rangle=\sin (\vartheta) \sin (\varphi)|a\rangle+\cos (\varphi)|e\rangle \\
& \quad+\cos (\vartheta) \sin (\varphi)\left|\phi_{C}\right\rangle, \\
& \left|\lambda_{-}^{\mathrm{T}}(t)\right\rangle=\sin (\vartheta) \cos (\varphi)|a\rangle-\sin (\varphi)|e\rangle \\
& \quad+\cos (\vartheta) \cos (\varphi)\left|\phi_{C}\right\rangle, \\
& \left|\lambda_{0}^{\mathrm{T}}(t)\right\rangle=\cos (\vartheta)|a\rangle-\sin (\vartheta)\left|\phi_{C}\right\rangle,
\end{aligned}
$$

where

$$
\begin{aligned}
& \tan \vartheta=\frac{\Omega_{p}(t)}{\Omega_{S}^{(C)}(t)}, \\
& \tan 2 \varphi=\frac{\Omega^{(C)}}{\Delta}, \\
& \Omega^{(C)}=\sqrt{\Omega_{P}^{2}(t)+\left(\Omega_{S}^{(C)}(t)\right)^{2}} .
\end{aligned}
$$

By considering the sa-STIRAP method in the three-state system, the following condition should be satisfied:

$$
H_{1}^{T}(t)=\mathrm{i} \hbar \sum_{n}\left|\partial_{t} \lambda_{n}^{\mathrm{T}}\right\rangle\left\langle\lambda_{n}^{\mathrm{T}}\right| .
$$

Now, considering in (28) that $\Omega_{a}^{(C)}=2 \dot{\vartheta}$, such (37) can be established that the additional transitions 
$|a\rangle \rightarrow|e\rangle$ and $\left|\phi_{C}\right\rangle \rightarrow|e\rangle$ will not be included in $(28)$. We can calculate $\dot{\vartheta}$ as follows:

$$
\dot{\vartheta}=\frac{\dot{\Omega}_{P}(t) \Omega_{S}^{(C)}(t)-\dot{\Omega}_{S}^{(C)}(t) \Omega_{P}(t)}{\left(\Omega^{(C)}\right)^{2}}
$$

such that

$$
\dot{\Omega}_{S}^{(C)}(t)=\frac{1}{\Omega_{S}^{(C)}(t)} \sum_{i=1}^{N}\left(\Omega_{S}^{(i)}(t) \dot{\Omega}_{S}^{(i)}(t)\right) .
$$

We impose that the initial state of the system is $|a\rangle$ but — just as it is in the three-level system the order of the pulses is counterintuitive (note that $\Omega_{S}^{C}(t)$ must precede $\left.\Omega_{P}(t)\right)$. Therefore, the reduced system will first be in the dark state $\left|\lambda_{0}^{\mathrm{T}}(t)\right\rangle$. When applying $H_{1}^{\mathrm{T}}(t)$, the system and the population will be completely transferred to a coherent superposition of other ground state $\left|\phi_{C}(t)\right\rangle$.

\section{Pulses design and numerical simulations}

We consider a situation, where the initial state of the system is $|a\rangle$. Our aim is to transfer the population from the initial state to a coherent superposition of states as follows:

$$
\left|\Psi\left(t_{f}\right)\right\rangle=\sum_{j=1}^{N} \alpha_{j}|j\rangle \quad \text { and } \quad \sum_{j=1}^{N}\left|\alpha_{j}\right|^{2}=1 .
$$

We design the pulses so that they satisfy the conditions (19) and the conditions of sa-STIRAP in the multi-level system. In order to perform the numerical study, we used the pump and Stokes pulses accordingly:

$$
\begin{aligned}
& \Omega_{P}(t)=\Omega_{0} \sin ^{4}\left(\pi \frac{(t-\tau)}{T}\right), \\
& \Omega_{S}^{(1)}(t)=\alpha_{1} \Omega_{0} \sin ^{4}\left(\pi \frac{t}{T}\right), \\
& \Omega_{S}^{(2)}(t)=\alpha_{2} \Omega_{0} \sin ^{4}\left(\pi \frac{t}{T}\right), \\
& \vdots \\
& \Omega_{S}^{(N)}(t)=\alpha_{N} \Omega_{0} \sin ^{4}\left(\pi \frac{t}{T}\right),
\end{aligned}
$$

where $\Omega_{0}$ is the peak value of pump Rabi frequency which can be adjusted in the experimental methods by the peak intensity of the pump laser pulse. Also, $\alpha_{i} \Omega_{0}(i=1,2, \ldots, N)$ are peak values of the Stokes-Rabi frequencies which can be adjusted using the peak intensity of the Stokes laser pulse. The additional pulses $\Omega_{a}^{(i)}(i=1,2, \ldots, N)$ should be designed in such a way as to match the sa-STIRAP conditions. We design these pulses as follows:

$$
\begin{gathered}
\Omega_{a}^{(1)}=2 \alpha_{1} \dot{\vartheta}, \\
\Omega_{a}^{(2)}=2 \alpha_{2} \dot{\vartheta}, \\
\vdots \\
\Omega_{a}^{(N)}=2 \alpha_{N} \dot{\vartheta}
\end{gathered}
$$
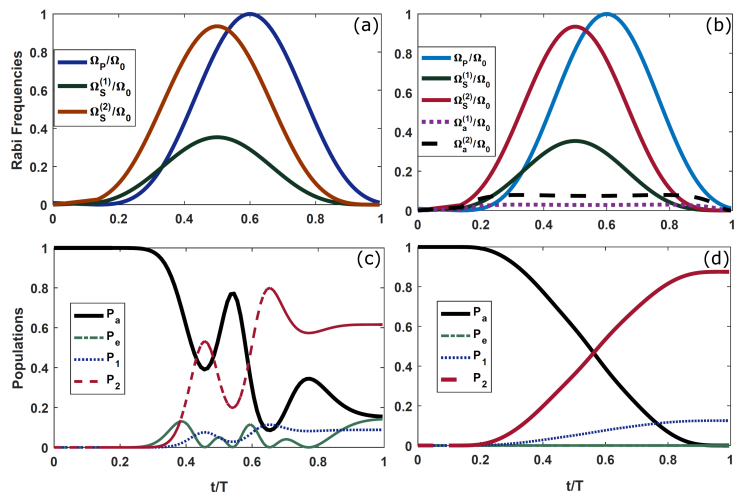

Fig. 4. Time evolution of the Rabi frequencies and time dependence of the $|a, e, 1,2\rangle$ populations in a multi-level system with $N=2$ for the STIRAP (a, c) and sa-STIRAP $(\mathrm{b}, \mathrm{d})$ methods, respectively, for $\Omega_{0}=2 \pi \times 2 \mathrm{MHz}, T=4 \mu \mathrm{s}, \Delta=0.2 \pi \mathrm{MHz}$ and $\tau=0.1 T$ in all cases.

Figure 4 shows an example for the superposition in the multi-state system with $N=2$ so that the desired state is $\left|\Psi_{\text {desired }}\right\rangle=\frac{1}{\sqrt{8}}|1\rangle+\sqrt{\frac{7}{8}}|2\rangle$. In Fig. 4a and $4 \mathrm{c}$, the time evolution of the Rabi frequencies and populations are plotted using the STIRAP pulse sequence, respectively, in which the adiabaticity breaks down and the population is not fully transferred into the desired state. In Fig. 4b and 4d, two additional fields, $\Omega_{a}^{(1)}(t)$ and $\Omega_{a}^{(2)}(t)$, are applied on the transitions of $|a\rangle \rightarrow|1\rangle$ and $|a\rangle \rightarrow|2\rangle$. In all cases, the pulses are designed using (41) and (42) and the laser parameters are the same. However, only in the case of Fig. 4d the full transfer of population to the final state can be seen.

\section{Robustness}

In Fig. 5, the final fidelity $F=\left|\left\langle\Psi\left(t_{f}\right) \mid \Psi_{\text {desired }}\right\rangle\right|^{2}$ of desired state $\left|\Psi_{\text {desired }}\right\rangle=\frac{1}{\sqrt{8}}|1\rangle+\sqrt{\frac{7}{8}}|2\rangle$ is plotted as a function of the time delay $\tau$ and the peak Rabi frequency $\Omega_{0}$ in the multi-level system with $N=2$ obtained with the sa-STIRAP method. The plateau region when $F=1$ corresponds to the region where the pulse shapes fulfill (41) and (42). As expected, the final fidelity of the desired state is not sensitive to different values of $\Omega_{0}$. As shown in Fig. 5 , there are two important limits for the pulse delay depending on $\Omega_{0}$ values, namely, a lower limit $(\tau \geq 0.08 T)$ and an upper limit $(\tau \leq 0.2 T)$ in which a complete population transfer occurs to the final desired state. The reason for these limits is that in this approach we use the STIRAP Stokes-pump pulse sequence.

In [45], it is shown analytically that there exists a lower and an upper limit for the pulse delay in the STIRAP pulse sequence. In the following, we study the effect of deviating from the calculated value for $\Omega_{a}^{(i)}(i=1,2, \ldots, N)$ on the final fidelity numerically. In real experiments, the values 


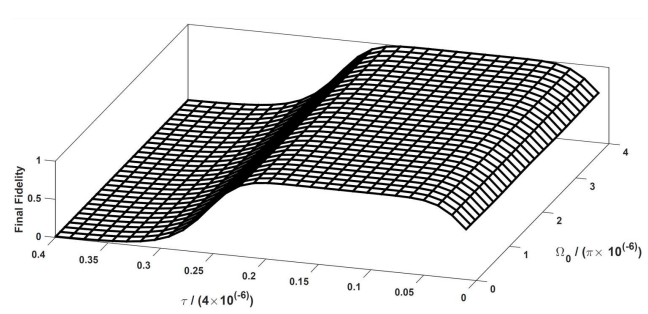

Fig. 5. Final fidelity of the desired state $\left|\Psi_{\text {desired }}\right\rangle$ is plotted against the time delay $\tau$ and the peak Rabi frequency $\Omega_{0}$ in a multi-level system with $N=$ 2 in resonance case $\Delta=0$. The pulse shapes are defined by (41) and (42). The other parameters are the same as in Fig. 4.

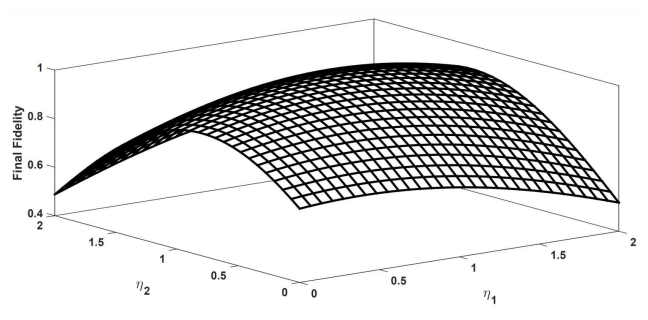

Fig. 6. The final fidelity of the desired state $\left|\Psi_{\text {desired }}\right\rangle$ is plotted against $\eta_{1}$ and $\eta_{2}$ in a multilevel system with $N=2$ in resonance case $\Delta=0$. The pulse shapes are defined by (41) and (43). The other parameters are the same as in Fig. 4.

obtained for $\Omega_{a}^{(i)}$ may differ from the values calculated in (43). In numerics, we assume that the $\Omega_{a}^{(i)}$ pulses are deviated by the $\eta_{i}$ parameter as follows:

$$
\begin{aligned}
& \Omega_{a}^{(1)}=2 \eta_{1} \alpha_{1} \dot{\vartheta}, \\
& \Omega_{a}^{(2)}=2 \eta_{2} \alpha_{2} \dot{\vartheta}, \\
& \vdots \\
& \Omega_{a}^{(N)}=2 \eta_{N} \alpha_{N} \dot{\vartheta} .
\end{aligned}
$$

It should be noted that the parameters $\eta_{J}$ used in the above equation are different from the parameters used in (19).

Figure 6 shows the final fidelity of the desired state $\left|\Psi_{\text {desired }}\right\rangle$ as a function of $\eta_{1}$ and $\eta_{2}$. As it can be observed, if the values of $\Omega_{a}^{(i)}$ are smaller or bigger than the values calculated with (42), the final fidelity will be affected in such a way that it will be less than one. According to Fig. 6 , if both the $\eta_{1}$ and $\eta_{2}$ values are equal to zero, it means that (i) the additional pulses have been removed and (ii) the technique has changed from sa-STIRAP to STIRAP. Due to the parameters used in this method, the population obtained in the STIRAP technique cannot be completely transferred to the desired state and so the final fidelity is much less than one. Also, if the $\eta_{1}$ and $\eta_{2}$ values are more than one, (i) the sa-STIRAP technique conditions will be destroyed and (ii) the final fidelity will be significantly reduced.

\section{Conclusion}

We have discussed the potential of the sa-STIRAP method for creating preselected coherent superpositions of states. As in STIRAP, the Stokes pulses arrive before the pump pulse but unlike STIRAP the initial populated ground state is paired to the final ground state using laser pulses different from the pump and Stokes pulses. We have suggested a smooth-pulse realization of the sa-STIRAP in multi-level systems which requires: a pump laser pulse, $N$ Stokes laser pulses and $N$ additional laser pulses that link the initial populated ground state to the final ground state. This method has similar properties to the threestate sa-STIRAP, regarding the robustness and efficiency and thus it can be considered as its analogue in creating coherent superpositions of states. We demonstrate that this method has a certain robustness with respect to finite fluctuations in the time delay and peak Rabi frequency.

\section{References}

[1] P. Král, I. Thanopulos, M. Shapiro, Rev. Mod. Phys. 79, 53 (2007).

[2] U. Gaubatz, P. Rudecki, S. Schiemann, K. Bergmann, J. Chem. Phys. 92, 5363 (1990).

[3] N.V. Vitanov, M. Fleischhauer, B.W. Shore, K. Bergmann, Adv. At. Mol. Opt. Phys. 46, 55 (2001).

[4] K. Bergmann, N.V. Vitanov, B.W. Shore, J. Chem. Phys. 142, 170901 (2015).

[5] N.V. Vitanov, A.A. Rangelov, B.W. Shore, K. Bergmann, Rev. Mod. Phys. 89, 015006 (2017).

[6] B.W. Shore, Adv. Opt. Photon. 9, 563 (2017).

[7] K. Bergmann, H.-Ch. Näger, C. Panda et al., J. Phys. B 52, 202001 (2019).

[8] B.W. Shore, The Theory of Coherent Atomic Excitation, Wiley, New York 1990.

[9] F. Vewinger, M. Heinz, R.G. Fernandez, N.V. Vitanov, K. Bergmann, Phys. Rev. Lett. 91, 213001 (2003).

[10] Z. Kis, N.V. Vitanov, A. Karpati, C. Barthel, K. Bergmann, Phys. Rev. A 72, 033403 (2005).

[11] M. Amniat-Talab, M. Saadati-Niari, S. Guérin, R. Nader-Ali, Phys. Rev. A 83, 013817 (2011).

[12] M. Amniat-Talab, M. Saadati-Niari, S. Guérin, Europ. Phys. J. D 66, 216 (2012).

[13] E.A. Shapiro, V. Milner, C. Menzel-Jones, M. Shapiro, Phys. Rev. Lett. 99, 033002 (2007). 
[14] S. Zhdanovich, E.A. Shapiro, M. Shapiro, J.W. Hepburn, V. Milner, Phys. Rev. Lett. 100, 103004 (2008).

[15] E.A. Shapiro, A. Peer, J. Ye, M. Shapiro, Phys. Rev. Lett. 101, 023601 (2008).

[16] A.A. Rangelov, N.V. Vitanov, Phys. Rev. A 85, 043407 (2012).

[17] J.A. Vaitkus, A.D. Greentree, Phys. Rev. A 87, 063820 (2013).

[18] M. Amniat-Talab, M. Saadati-Niari, Europ. Phys. J. D 69, 215 (2015).

[19] M. Saadati-Niari, Ann. Phys. 372, 138 (2016).

[20] B. Nedaee-Shakarab, M. Saadati-Niari, F. Zolfagharpour, Phys. Rev. C 96, 044619 (2017).

[21] S. Mirza-Zadeh, M. Saadati-Niari, M. Amniat-Talab, Laser Phys. Lett. 15, 095105 (2018).

[22] N. Irani, M. Saadati-Niari, M. AmniatTalab, Phys. Scr. 95, 035109 (2020).

[23] A. Ruschhaupt, X. Chen, D. Alonso, J.G. Muga, New J.Phys. 14, 093040 (2012).

[24] R.G. Unanyan, L.P. Yatsenko, K. Bergmann, B.W. Shore, Opt. Commun. 139, 48 (1997).

[25] M. Demirplak, S.A. Rice, J. Phys. Chem. A 107, 9937 (2003).

[26] M. Demirplak, S.A. Rice, J. Chem. Phys. 129, 15411 (2008).

[27] X. Chen, I. Lizuain, A. Ruschhaupt, D. Guéry-Odelin, J.G. Muga, Phys. Rev. Lett. 105, 123003 (2010).

[28] S. Masuda, K. Nakamura, Proc. R. Soc. Lond. A 466, 1135 (2010).
[29] M.B. Berry, J. Phys. A Math. Theor. 42, 365303 (2009).

[30] X. Chen, E. Torrontegui, J.G. Muga, Phys. Rev. A 83, 062116 (2011).

[31] X. Chen, J.G. Muga, Phys. Rev. A 86 , 033405 (2012).

[32] M. Lu, Y. Xia, L.T. Shen, J. Song, N.B. An, Phys. Rev. A 89, 012326 (2014).

[33] A. del Campo, Phys. Rev. Lett. 111, 100502 (2013).

[34] A. del Campo, Phys. Rev. A 84, 031606(R) (2011).

[35] A. del Campo, M.G. Boshier, Sci. Rep. 2 , 648 (2012).

[36] Y.H. Chen, Y. Xia, Q.Q. Chen, J. Song, Phys. Rev. A 89, 033856(2014).

[37] T.Y. Lin, F.C. Hsiao, Y.W. Jhang, C. Hu, S.Y. Tseng, Opt. Expr. 20, 24085 (2012).

[38] K. Paul, A.K. Sarma, Phys. Rev. A 91, 053406 (2015).

[39] Y.H. Chen, Y. Xia, J. Song, Q.Q. Chen, Sci. Rep. 5, 15616 (2015).

[40] K. Bergmann, H. Theuer, B.W. Shore, Rev. Mod. Phys. 70, 1003 (1998).

[41] M. Fewell, B. Shore, K. Bergmann, Austr. J. Phys. 50, 281 (1997).

[42] J.R. Morris, B.W. Shore, Phys. Rev. A 27, 906 (1983).

[43] A.A. Rangelov, N.V. Vitanov, B.W. Shore, Phys. Rev. A 74, 053402 (2006).

[44] B.W. Shore, J. Mod. Opt. 61, 787 (2014).

[45] N.V. Vitanov, K.A. Suominen, B.W. Shore, J. Phys. B 32, 4535 (1999). 